\title{
The impact of trading volume, number of trades and overnight returns on forecasting the daily realized range
}

\author{
${ }^{a}$ Neda Todorova \\ Corresponding author. \\ Griffith Business School, Griffith University \\ 170 Kessels Road, Nathan, Queensland 4111, Australia \\ Phone: +6173735 7219, Fax: +6173735 3719 \\ Email: n.todorova@griffith.edu.au \\ ${ }^{b}$ Michael Souček \\ Department of Business Administration, \\ European University Viadrina \\ Große Scharrnstraße 59, D-15230 Frankfurt (Oder), Germany \\ Email: soucek@europa-uni.de
}

\begin{abstract}
Intraday data of 26 German stocks are used to investigate whether the information contained in trading volume and number of trades as well as in various specifications of overnight returns can improve one-step-ahead volatility forecasts. For this purpose, a HAR model of the realized range adjusted for discrete trading is augmented by each of these variables and compared with the model's default form. The results show that the considered liquidity measures lead to very modest improvements in forecasting performance. The overnight returns exhibit some in-sample forecasting power. However, the accuracy improvement of out-of-sample forecasts is unequivocally non-significant.
\end{abstract}

Keywords: Volatility forecasting; HAR model; Realized range; Overnight returns; Trading volume; Number of trades.

JEL Classification: C13; C14; C22; C53; G17 


\section{Introduction}

Financial market volatility enjoys a sustained interest in theoretical and empirical literature. Over time, the increasing availability of transaction and quote data has allowed the focus to be set on intraday levels. High-frequency data enable the application of advanced volatility proxies like the realized volatility based on summing squared intraday returns (Andersen and Bollerslev, 1998) or the realized range comprising the sum of scaled intraday price ranges (Christensen and Podolskij, 2007; Martens and van Dijk, 2007). Among others, issues of current research interest are, the development of advanced forecasting approaches (Corsi, 2009; Ghysels et al., 2006), the adjustment of existing estimators in order to deal with empirical drawbacks (Zhang et al., 2005; Hansen and Lunde, 2006) as well as testing and accounting for the existence of jumps (Andersen et al., 2007).

The aim of this study is to examine whether in-sample and out-of-sample forecasting improvements can be achieved by augmenting a heterogeneous autoregressive (HAR) model for the realized range with common liquidity measures and various specifications of overnight returns. Due to its easy application and overall very good forecasting performance, the HAR model is broadly supported in the plethora of modelling and forecasting approaches for financial markets volatility.

This article complements the large body of literature in several directions. First, the incremental economic value of trading volume, number of daily transactions and of overnight returns for forecasting return volatility is analyzed for a large stock sample. The research is motivated by the pronounced lead-lag correlations of these variables to the daily volatility. Second, the results are sorted by the stocks' liquidity level in order to enhance insights into possible forecast improvements. Third, since the realized range established by Martens and van Dijk (2007) and Christensen and Podolskij (2007) is used instead of realized volatility, as in the original model proposed by Corsi (2009), the study also demonstrates its eligibility for accurate volatility modelling within a (augmented) HAR model.

The notion that liquidity measures like trading volume, number of transactions, bid-ask spreads, or overall market liquidity are related to the return volatility is widespread. A number 
of studies address the relationship between volume and return volatility. A popular theoretical explanation is based on the mixture of distribution hypothesis which suggests that volatility is positively related to trading volume due to its dependence on a common latent mixing variable, the rate of information arrival (Park, 2010). However, a consensus on this relationship and its economic significance has not been reached yet. Chen et al. (2001) investigate index volatility of nine markets and conclude that trading volume contributes some information to the process of equity index returns. Darrat et al. (2003) find evidence of significant lead-lag relations between trading volume and return volatility in a large number of the DJIA stocks. Considering trading volume as a proxy for the changes in the information set available to market participants, Donaldson and Kamstra (2005) show that trading volume has a switching role between volatility forecasts based on stale information and option-implied estimates. Fuertes et al. (2009) also utilize trading volume for assigning market conditions for volatility forecasting. However, it is not certain whether the volume's informational role can be exploited to enhance performance when volume is explicitly incorporated into forecasting models. Brooks (1998) generates GARCH forecasts based on daily data and shows that augmenting daily stock return volatility models with measures of lagged volume leads only to very modest improvements, in terms of forecasting performance. ${ }^{1}$

Fleming and Kirby (2011) suggest that return volatility and trading volume exhibit similar long-run dynamics and include the logarithms of volatility and volume into a trend-stationary fractionally-integrated process. Their results show only minor gains for improving short-term volatility forecasts. We address this issue by incorporating trading volume information into another economically plausible volatility model with an advanced non-parametric volatility estimator and utilizing an extensive data set from the German market.

Taking standard liquidity measures into account, Jones et al. (1994) argue that the positive volatility-volume relation actually reflects the positive relation between volatility and the number of transactions, concluding that volatility is generated by the frequency of transactions, and not by their size. Thus, trading volume carries no information beyond that contained

\footnotetext{
${ }^{1}$ See also Andersen (1996) and Bollerslev and Jubinski (1999) for a literature review of older studies.
} 
in the frequency of transactions. Naes and Skjeltorp (2006) present empirical evidence from order data of the Oslo Stock Exchange and also confirm that the number of trades is a more meaningful measure for investigating the relation between volatility and liquidity. Chan and Fong (2000) examine the number and size of trades, as well as order imbalances and provide evidence of their significant role in the volatility-volume relation for a sample of NYSE and Nasdaq stocks. In this context, the incorporation of the number of trades apart from the daily transaction volume appears to be a promising attempt to enhance forecasting accuracy.

Aiming to explain the persistence of volatility established by using GARCH-type models for 10 actively traded US stocks, Gallo and Pacini (2000) conclude that the information about the trading activity of the previous day is inferior to the information which arrives during the time when the market is closed. Taking overnight jumps into account when estimating multiday volatility is an important issue especially for practical purposes. There is a number of studies proposing different ways to include the impact of information arrivals during the closed-market time in the context of realized variance (e.g. Martens, 2002; Fleming et al., 2003; Koopman et al., 2004; Hansen and Lunde, 2005). Hansen and Lunde (2005) argue that, despite being very noisy, overnight returns do contain useful information beyond that included in realized volatility gained by returns observed during open market time. Using the functional coefficient model of Cai et al. (2000), Gallo (2001) provides some significant in-sample evidence of the impact of overnight surprises on intraday returns. However, the overnight surprises are found not to contribute to a significant improvements of out-of-sample volatility forecasts. On the other hand, Tseng et al. (2012) investigate three stock indices on the Taiwan Stock Exchange incorporating the previous nights' absolute returns into a HAR model. Their findings indicate that taking information arrivals from the market's nontrading time into account improves the performance of volatility forecasts in both in-sample and out-of-sample analysis.

Overall, these findings leave the question of the statistical significance of the impact of overnight returns and liquidity measures for volatility forecasting not conclusively clarified. The current study adopts a similar approach like Tseng et al. (2012) but runs an empirical analysis for a substantially larger data base, a different sample period and another asset class, 
investigating liquidity measures as well as various specifications of the overnight returns.

The article is arranged as follows. The next section presents the methodology of the study. Subsequent sections describe the data, in-sample and out-of-sample results. The final section concludes.

\section{Methodology}

\subsection{Volatility proxy}

The sum of squared intraday returns which is known in the recent literature as realized variance (often also referred to as realized volatility) firstly proposed by Andersen and Bollerslev (1998) is currently the most adopted estimator of integrated variance of one day in discrete settings. Andersen et al. (2003) show that the realized variance which uses all available data is a consistent estimator of the integrated variance when there is no microstructure noise. The increasing availability of high-frequency data and the theoretical properties of realized volatility make this estimator very appealing. However, very high-frequency prices are heavily contaminated by market microstructure effects, such as the bid-ask bounce, which distort the realized volatility to an extent dependent on the properties of the noise. The bias caused by microstructure noise is subject of active research interest (e.g., Bandi and Russell, 2006; Hansen and Lunde, 2006, among others). Various ways of dealing with the distortion due to microstructure noise are possible. A simple, often adopted solution of this problem is to sample at lower frequencies, for example every 5 minutes. Alternatively, several bias correction procedures have been proposed, such as the subsampling and the kernel-based approaches. ${ }^{2}$

Apart from the broadly accepted return-based concepts for modelling financial market volatility, a separate strand of literature focuses on the estimation of an asset's volatility from its high-low trading ranges. These estimators rely on the notion that high-low price ranges contain more information than the corresponding returns as a range arises from the entire price process, whereas the return is calculated from price observations sampled at fixed points in

\footnotetext{
${ }^{2}$ A summary of basic assumptions about microstructure noise, their implications for realized volatility as well as various adjustment approaches can be found in McAleer and Medeiros (2008).
} 
time. So, a properly scaled price range is expected to capture financial market volatility better than the respective returns. Especially since advanced volatility proxies based on intraday data are used as a volatility benchmark, the efficiency of price ranges is broadly recognized in the literature (Shu and Zhang, 2006; Jacob and Vipul, 2008; Todorova and Husmann, 2012; Todorova, 2012).

Until 2007, the established extreme-value volatility estimators accounted only for daily price ranges without breaking the computation down to intraday levels. Encouraged by the efficiency of the daily ranges, Martens and van Dijk (2007) and Christensen and Podolskij (2007) combine the concepts of range-based and scarcely sampled realized volatility. Specifically, they suggest to measure daily volatility using the sum of high-low Parkinson (1980) ranges for non-overlapping intraday intervals of a fixed length. This is the so-called realized range

$$
R R_{t}=\sqrt{\frac{1}{4 \ln 2} \sum_{i=1}^{I}\left(H_{t, i}-L_{t, i}\right)^{2}}
$$

where $I$ is the number of intraday intervals and $H_{t, i}$ and $L_{t, i}$ denote the highest and lowest log price observations in the $i$-th intraday interval of day $t$, respectively. Todorova and Husmann (2012) compare the empirical performance of the realized range as applied to modelling stock return volatility and establish that the realized range is much more precise than daily ranges. The main determinant of its empirical performance for moderate levels of stock liquidity appears to be the number of available observations because range-based estimators are derived under the assumption of continuous trading and are expected to exhibit a downward bias due to the discreteness of the price process. The correction procedure of Christensen and Podolskij (2007), which accounts particularly for this drawback, proves to work very well. The main idea of this adjustment is to replace the factor $(4 \ln 2)$ by $\lambda_{2, m}$, which is the second moment of the range of a standard Brownian motion over a unit interval when the price path is discrete and $m$ increments of the underlying continuous process are available.

Since our object of interest is the latent volatility process, measurement errors must be taken into account in the estimation. Following Todorova and Husmann (2012), we adjust 
the realized range according to the approach of Christensen and Podolskij (2007). However, the theoretical results of Christensen and Podolskij (2007) are based on the assumption of equidistant price observations which is obviously not the case for the available stock transaction data. A correction of the realized range for irregular sampling is proposed by Rossi and Spazzini (2009). However, caution is necessary when applying this procedure due to the numerical instability of the scaling factor for intervals with very few price observations. In this study, the sum of the intraday ranges is corrected according to the procedure of Christensen and Podolskij (2007) but are rather weighted by a factor $\lambda_{2, n}$, with $n$ denoting the average number of price observations of an intraday interval. $\lambda_{2, n}$ is individually determined for every day in the sample. To estimate $\lambda_{2, n}$, a Brownian motion is simulated with 1000000 replications:

$$
B_{i}=B_{i-1}+\frac{1}{\sqrt{n}} \epsilon_{i}, \quad i=1, \ldots . n, \epsilon_{i} \sim N(0,1) .
$$

Table 1 provides summary statistics of the realized range in terms of annualized volatilities (computed as daily volatility multiplied by $\sqrt{252}$ ), due to its ease of interpreting magnitudes. ${ }^{3}$ These volatility measures are obtained by sampling every 10 minutes.

The descriptive statistics confirm that daily volatility measures are characterized by a notable time series variation. Furthermore, the realized range exhibits positive skewness and positive excess kurtosis for all equities and is thus in line with documented stylized facts of financial market volatility.

\subsection{Volatility modelling technique}

Motivated by the heterogeneous market hypothesis presented by Müller et al. (1997) which recognizes the presence of heterogeneity across traders, Corsi (2009) proposes a simple autoregressive-type model of realized volatility, the HAR model, which incorporates volatilities realized over different interval sizes. The main idea is that traders with different time horizons cause different types of volatility components. This notion is supported by the observation that volatility over longer time intervals has a stronger influence on volatility over shorter time

\footnotetext{
${ }^{3}$ The stocks are sorted by liquidity. For details, see the next section.
} 
intervals than vice versa. In the default version of this cascade-like consideration, volatility forecasts are linear functions of the current daily, weekly, and monthly realized volatilities. The HAR model successfully captures the long-memory feature of realized variance for various forecasting horizons and is used in numerous studies on financial volatility (Andersen et al., 2007; Ait-Sahalia and Mancini, 2008; McAleer and Medeiros, 2008; Tseng et al., 2012, among others). Corsi (2009) models daily volality in terms of the square root of the realized variance. Since the realized range of Martens and van Dijk (2007) and Christensen and Podolskij (2007) proves to be a highly efficient volatility estimator, the realized volatility in the original HAR model is substituted by the realized range, similarly to Tseng et al. (2012). We maintain the original form of Corsi (2009) and consider the square roots of the sums of intraday ranges,

$$
R R_{t}=\alpha+\beta_{1} R R_{t-1}+\beta_{2} R R_{t-5, t-1}+\beta_{3} R R_{t-21, t-1}+\omega_{t}
$$

The multiday volatility measures $R R_{t-5, t-1}$ and $R R_{t-21, t-1}$ are established by taking the mean of the daily volatilities over the relevant time period. Furthermore, to investigate the role of the number of transactions, daily volume and overnight returns for forecasting one-step-ahead daily volatility, the HAR model is extended to

$$
R R_{t}=\alpha+\beta_{1} R R_{t-1}+\beta_{2} R R_{t-5, t-1}+\beta_{3} R R_{t-21, t-1}+\beta_{4} X_{t-1}+\omega_{t}
$$

with $X_{t-1}$ being the lagged additional variable.

Regarding the specification of the overnight returns between days $t-1$ and $t$, we consider in particular the overnight return $R_{t-1, t}$ defined as the difference of the logs of the open price of day $t$ and the closing price of the previous day multiplied by 100. Additionally, this return is used in absolute terms, $R A B S_{t-1, t}$. Last, a leverage effect is taken into account by considering overnight returns in the cases when they are negative and setting them equal to zero otherwise. This specification is referred to as $R N E G_{t-1, t}$.

The notion of augmenting an established volatility model is broadly used in the literature on financial volatility. Specifically, the HAR model has already been extended to contain jump 
variables (Andersen et al., 2007), leverage effects (Corsi and Renó, 2012) and absolute overnight returns (Tseng et al., 2012). In this study, the extended HAR model (equation (4)) is used in the in-sample analysis and for generating out-of-sample one-step-ahead forecasts.

\section{Data}

The analysis is based on transaction data from XETRA of 26 constituents of the leading German index DAX 30. The DAX stocks are the most actively traded German equities. The data were supplied by the Karlsruher Kapitalmarktdatenbank. The sample period extends from January 1, 2003, until December 31, 2007, for a total of 1268 trading days. The daily transaction records extend from 9:00 am to 8:00 pm until October 31, 2003 and to 5:30 pm thereafter. Thus, 66 10-minute intervals cover the eleven hours of open market time from January 2003 to October 2003. From November 2003 onwards, the trading closes at $5.30 \mathrm{pm}$ so that 51 10-minute intervals are available covering the 8.5 hours open market time.

Generally, the change of the trading day's length may be a source of a bias. However, since the study aims at identifying the incremental role of various variables beyond the informational content of daily volatility, and not at the unbiasedness of volatility forecasts which might be negatively influenced by such a distortion, we feel that removing of prices after 5:30 pm for the first ten months of the sample period would relocate informational content from the realized range to the subsequent overnight returns. Since our aim is to explore exactly the impact of overnight returns on intraday volatility, a larger bias is expected to arise from any adjustment of the trading time. Moreover, discarding data over the last two and a half hours trading time is also likely to cause a severe contamination of the data. Aiming to assess volatility modelling under real-life market conditions, we refrain from adjusting the relevant intervals of trading time.

The raw transaction data are filtered for outliers, for prices without assigned transaction volume and transactions beyond the official closing time of XETRA. Furthermore, stock splits are observed for several of the stocks during the period under consideration. Since the stock splits occur overnight, the involved overnight returns are set to zero. Generally, stock splits 
may also give rise to biases in terms of daily volume. Because a thorough investigation of the volume values around these events did not identify consistent strong enhancements of the daily volume in proportions equaling the ratio of the face value splits, we are aware of this potential bias but disregard an adjustment due to its potential arbitrariness.

Table 2 lists basic characteristics of the variables analyzed for all considered stocks sorted by liquidity. The overnight returns are computed by utilizing the last trading price observed before the end of the trading day and the first price documented for the next day. The daily volume $V O L$ corresponds to the number of traded stocks during the open market time summed over all trades. NTR denotes the number of transactions per one day. Since the daily volume depends on the face value of the underlying stocks, the number of daily trades is considered to be a more reliable measure of stock liquidity. That is why the equities are sorted by NTR . The tabulated values show that all variables exhibit a high variation over time. The sample period comprises periods of high and low liquidity and volatile overnight returns. For individual stocks, absolute overnight returns of up to $9 \%$ are observed.

To assess the plausibility of utilizing $V O L, N T R$ and the overnight returns for modelling stock volatility, correlations between the realized range of day $t$, the daily volume and the number of trades on day $t-1$ and the immediately preceding overnight return are presented in table 3. Significant correlations would be a sign of the existence of an informational content potentially useful for explaining the variation of daily volatilities.

$\operatorname{Cor}\left(R R_{t}, R_{t-1, t}\right)$ is most weakly pronounced, as expected, and exhibits significant values mostly for comparatively more liquid stocks. The correlations between the realized range to $R A B S$ and $R N E G$ are significant at the $1 \%$ level without exception. While $R A B S$ is consistently negatively correlated with the open-market time volatility of the next day, $R N E G$ exhibits positive values for all stocks. Finally, the absolute values of $\operatorname{Cor}\left(R R_{t}, R A B S_{t-1, t}\right)$ are lower than these of $\operatorname{Cor}\left(R R_{t}, R N E G_{t-1, t}\right)$ confirming the relevance of a leverage effect in regard to financial assets' volatility.

The correlations of the realized range to the measures $V O L$ and $N T R$ of the preceding days are positive with two exceptions (Deutsche Post for $V O L$ and RWE for NTR). However, 
$\operatorname{Cor}\left(R R_{t}, V O L_{t-1}\right)$ and $\operatorname{Cor}\left(R R_{t}, N T R_{t-1}\right)$ are not so consistently identified across all stocks. Also noteworthy in this context is that the non-significant values of $\operatorname{Cor}\left(R R_{t}, V O L_{t-1}\right)$ do not relate to the cases of observed stock splits. In general, the correlations are more consistently statistically significant for stocks of higher liquidity. ${ }^{4}$

Overall, the correlation analysis suggests that the considered variables exhibit some informational content about the future variation of stock returns. The following empirical results shed light on the question whether this information is incremental to that already contained in historical daily volatilities.

\section{Results}

\subsection{In-sample results}

For assessing the informational content of the presented variables for forecasting purposes, the original HAR model (3) and the extended HAR models (4) utilizing $R_{t-1, t}, R A B S_{t-1, t}$, $R N E G_{t-1, t}, V O L_{t-1}$ and $N T R_{t-1}$ as the additional variable $X_{t-1}$, are run for all 26 stocks. The in-sample analysis comprises the period from 2003 to 2006, with the first 21 days necessary to establish $R R_{t-21, t-1}$. $V O L_{t}$ is scaled by dividing the daily volume by 1000000 . Analogously, $N T R_{t}$ is divided by 10000 .

The estimation results with Newey-West standard errors are provided in table 4 . The results are presented in aggregated form in order to save space. The six panels are identically constructed. The columns Mean, SD, Min und Max present the coefficients' descriptive statistics of all 26 values within the relevant model. The columns labelled \#5\% and \#1\% show in how many out of the 26 considered cases the coefficients are significant at the $5 \%$ and $1 \%$ level, respectively.

The parameters weighting the past observations of daily volatility are consistently positive and clearly identified for all models. The estimates of $\beta_{1}$ and $\beta_{2}$ are for the most part significant at the $1 \%$ level and without exception significant at the $5 \%$ level. In up to two cases for each

\footnotetext{
${ }^{4}$ In contrast, the contemporaneous correlations $\operatorname{Cor}\left(R R_{t}, V O L_{t}\right)$ and $\operatorname{Cor}\left(R R_{t}, N T R_{t}\right)$ (not tabulated) are all positive and invariably statistically significant at the $1 \%$ level. However, contemporaneous interrelations are less meaningful for forecasting purposes.
} 
model, $\beta_{3}$ has a $p$-value higher than 0.05 . The highest $p$-value $(0.1287)$ occurs for the HAR-NTRmodel. Furthermore, the magnitude of the parameter estimates indicates that the volatility over the last week has the highest relative importance for modelling one-step-ahead volatilities. Overall, the existence of highly persistent volatility dependence is confirmed.

Extending the one-step-ahead HAR model by daily volume and number of trades leads to unequivocal results. The estimated coefficients for these variables do not exhibit uniform signs and are in none of the cases significant at the $5 \%$ level. The mean $p$-values with $0.5394(V O L)$ and $0.4969(N T R)$ are very high. In contrast, the coefficients $\beta_{1}, \beta_{2}$ and $\beta_{3}$ remain significant. These results indicate that the daily volume and the number of trades are not likely to contain substantial incremental information beyond that of past volatility values as considered by a cascade-like autoregressive model for a forecasting horizon of one day. This striking lack of explanatory power of the number of trades and transaction volume is somehow surprising in the context of the broadly significant lead-lag correlations as described above. However, it is consistent with the results of some extant studies on the stocks' trading volume (Brooks, 1998; Fuertes et al., 2009).

Regarding the informational content of overnight returns, the results are more heterogeneous. It becomes obvious that the overnight jump contains some information since in 9 to 12 cases, depending on the exact specification of the overnight returns, the coefficients are significant at the $5 \%$ level. Looking at the variation of the $p$-values more closely indicates that $R N E G$ exhibits comparatively the most stable behavior. The highest number of significant estimates is observed when considering negative values and dropping positive values of the overnight jumps. Furthermore, consistent with the expected leverage effect, the signs of all coefficients are negative.

In order to assess the potential improvement of the forecasting performance of the realized range by incorporating further variables, the adjusted $R^{2}$ of the HAR models are compared to the adjusted $R^{2}$ of the default HAR model which disregards the additional components (table 5). An interesting general observation is that the overall explanatory power of the HAR models seems to increase with higher stock liquidity which is consistent with the properties 
of the realized range established by Todorova and Husmann (2012). However, the explicit consideration of liquidity measures leads to very modest improvements only. In none of these cases, the adjusted $R^{2}$ is enhanced by more than 0.005 . In contrast, the overnight returns have a stronger explanatory power. It can be again confirmed, that the overnight return $R$ is inferior to the absolute return $R A B S$ and the leverage effect established by $R N E G$. Incorporating $R A B S$ leads in 3 cases to improvement of more than 0.01 and in 8 cases to an enhancement of the adjusted $R^{2}$ by 0.005 to 0.01 . The coefficient of determination increases by more than 0.01 in 4 cases due to RNEG and for 7 stocks by an amount between 0.005 and 0.01 .

Overall, the findings suggest that the realized range may be affected by overnight jumps. However, the comparison of our results with Tseng et al. (2012) supposes that the degree of this impact appears to be less pronounced for our sample.

Finally, the performance of the specifications $R A B S$ and $R N E G$ is compared by encompassing both variables in the regression

$$
R R_{t}=\alpha+\beta_{1} R R_{t-1}+\beta_{2} R R_{t-5, t-1}+\beta_{3} R R_{t-21, t-1}+\beta_{4} R N E G_{t-1, t}+\beta_{5} R A B S_{t-1, t}+\omega_{t}
$$

and assessing the statistical significance of the parameters $\beta_{4}$ and $\beta_{5}$. The results are summarized in table 6 . Considering both specifications together does not lead to a notable increase of the adjusted $R^{2}$ arising from the HAR-RABS und HAR-RNEG models in table 5. This confirms that $R A B S$ and $R N E G$ contain very similar information. A strong conclusion in favor of one of the specifications is difficult. It appears that $R A B S$ is significant in more cases for less liquid stocks whereas the impact of $R N E G$ is prevailing for stocks of relatively higher number of trades.

Generally, since liquidity measures from the open market time and price changes during closed market time may contain different information, additional insights might be gained by incorporating a liquidity and an overnight jump indicator into a HAR model. However, due to the conclusive lack of in-sample forecasting power of the considered liquidity measures, the analysis of encompassing volatility models seems redundant. 


\subsection{Out-of-sample results}

The in-sample results show that augmenting a HAR model for the one-step ahead realized range with information about the daily stock's volume and number of trades is hardly expected to increase its predictive capability. For information arrivals during nontrading periods, the findings are ambiguous.

For consistency, all in-sample models are used to generate one-step-ahead forecasts applying a rolling window approach for the last 1000 observations of the realized range of the last day, week and month. ${ }^{5}$ Since the first 21 days are necessary for establishing $R R_{t-21, t-1}, 248$ forecasts for the period January 5, 2007 to December 27, 2007 are obtained.

The results presented in table 7 clearly reveal that the additional variables actually lead to lower mean squared errors (MSE) than the default form of the HAR model in almost all cases. ${ }^{6}$ However, this does not necessarily mean that the improvement is significantly different from zero. In order to test whether the mean squared errors statistically differ from each other, the Diebold-Mariano (DM) one-sided test (Diebold and Mariano, 1995) examining whether the relevant augmented HAR model achieves mean squared errors which are significantly higher than or equal to these arising from the default HAR model is used. Table 7 contains the $p$-values of the these DM tests. It is immediately obvious that none of the augmented HAR models is able to provide significantly better one-step-ahead volatility forecasts. Since these forecasts exhibit lower $p$-values, the superiority of the overnight returns especially in the $R A B S$ and $R N E G$ specification towards the other additional variables is also confirmed. However, their magnitude is by far not sufficient to reject the DM test.

These results comply with the conclusions of Gallo (2001) and are at odds with the study of Tseng et al. (2012) where the HAR models containing the absolute overnight return provide, at least at the $10 \%$ level, significantly better volatility forecasts than the default HAR model. A basic point of reference for interpreting the differences between the results is the fact that the trading day in the study of Tseng et al. (2012) comprises 4.5 hours per day whereas the

\footnotetext{
${ }^{5}$ Strictly speaking, by including information on the overnight returns the forecasts (for models including $R$, $R N E G$ or $R A B S$ ) can only be done once the first price of the new day has been established.

${ }^{6}$ The MSE values are multiplied by $10^{3}$ for clarity.
} 
daily volatility in our study is gained from open market time of 11 hours till November 2003 and 8.5 hours thereafter. Therefore, besides being noisy, the overnight returns might be less informative because they account for informational arrivals during shorter periods of time.

\subsection{Robustness of the results}

To assess the robustness of our findings gained across the 26 considered equities, additional checks are conducted. The results are qualitatively the same and are omitted for brevity but are available upon request. First, the analysis is repeated with the realized range established by sampling at 5 and 15 minutes intervals. Furthermore, the in-sample analysis is extended by encompassing up to five lags of the additional variables in equation (4). The estimation coefficients $\beta_{1}, \beta_{2}$ and $\beta_{3}$ of the standard HAR model remain highly significant and similar to those presented in tables 4 and 6 . While the lagged indicators of trade size and number of transactions remain consistently insignificant, the coefficients of past overnight returns, especially in the absolute and leveraged specifications, are significant, at least at the $10 \%$ level, in a few cases. However, if any, these estimation results give rise to only negligible $R^{2}$ enhancements. Additionally, the regression analysis for the in-sample period as well as for the whole sample is repeated by using the variance specification of the volatility proxy $\left(R R^{2}\right)$. This consideration does not yield insights contradicting the already discussed findings.

\section{Conclusion}

This study investigates whether standard liquidity measures like the daily volume and the number of transactions as well as different specifications of overnight returns exhibit incremental informational content when modelling one-step-ahead forecasts with a HAR model. The analysis is motivated by the pronounced, highly significant lead-lag correlations between the utilized measure of daily volatility and these variables. As a proxy of daily volatility, the realized range corrected according to the approach of Christensen and Podolskij (2007) is adopted. The analysis comprises 5 years of transaction data for 26 German stocks.

The analysis makes the following points. For in-sample forecasting, the overnight returns exhibit some forecasting power for a number of equities, while the evidence that the considered 
liquidity measures are not capable of improving forecast accuracy is quite strong. For all assets, the estimation coefficients of the daily volume and number of transactions are insignificant at conventional levels of significance. In respect to the question which is the most appropriate specification of the overnight returns, the absolute returns seem to be more feasible for less liquid stocks whereas a leveraged overnight return achieves better results for stocks of comparatively higher number of daily transactions.

Regarding out-of-sample forecasting, the augmented HAR models provide lower mean squared errors in most of the cases. However, it becomes evident that none of the additional variables is able to contribute significantly to a forecasting improvement. This holds without exception for all stocks indicating that incorporation of liquidity measures and overnight returns is dispensable for these assets and the period under consideration.

The results comply with extant literature on the marginal incremental value of transaction volume for modelling financial markets volatility. Additionally, evidence is provided that the information inherent in the daily number of trades cannot be exploited within the adopted methodology to achieve a significantly better in-sample performance and out-of-sample forecasts. Regarding the informational content of overnight returns, our results are at odds with the study of Tseng et al. (2012) probably due to the different lengths of the open-market time and are more in line with Gallo (2001). Overnight returns are hence to be investigated for further periods, assets and forecasting horizons. Furthermore, it is possible that there are holidays and day-of-the-week effects in volatility which could be extracted and exploited for increasing forecasting accuracy. In this context, the separate consideration of jumps over weekends and holidays might shed further light on the informational content of overnight returns. 


\section{References}

Ait-Sahalia, Y., Mancini, L., 2008. Out of sample forecasts of quadratic variation. J. Econometrics 147, 17-33.

Andersen, T., 1996. Return volatility and trading volume: An information flow interpretation of stochastic volatility. J. Finance 51, 169-204.

Andersen, T.G., Bollerslev, T., 1998. Answering the skeptics: Yes, standard volatility models do provide accurate forecasts. Int. Econ. Rev. 39, 885-905.

Andersen, T.G., Bollerslev, T., Diebold, F.X., Labys, P., 2003. Modeling and forecasting realized volatility. Econometrica 71, 579-625.

Andersen, T.G., Bollerslev, T., Diebold, F.X., 2007. Roughing it up: Including jump components in the measurement, modeling and forecasting of return volatility. Rev. Econ. Stat. 89, $701-720$.

Bandi, F., Russell, J.R., 2006. Separating microstructure noise from volatility. J. Fin. Econ. 79, 655-692.

Bollerslev, T., Jubinski, D., 1999. Equity trading volume and volatility: Latent information arrivals and common long-run dependencies. J. Bus. Econ. Stat. 17, 9-21.

Brooks, C., 1998. Predicting stock index volatility: Can market volume help? J. Forecast. 17, $59-80$.

Cai, Z., Fan, J., Yao, Q., 2000. Functional-coefficient regression models for nonlinear time series. J. Amer. Statist. Assoc. 95, 941-956.

Chan, K., Fong, W.-M., 2000. Trade size, order imbalance, and the volatility-volume relation. J. Fin. Econ. 57, 247-273.

Chen, G.-M., Firth M., Rui, O. M., 2001. The dynamic relation between stock returns, trading volume, and volatility. Fin. Rev. 36, 153-174. 
Christensen, K., Podolskij, M., 2007. Realized range-based estimation of integrated variance. J. Econometrics 141, 323-349.

Corsi, F., 2009. A simple approximate long-memory model of realized volatility. J. Fin. Econometrics 7, 174-196.

Corsi F., Renó R., 2012. Discrete-time volatility forecasting with persistent leverage effect and the link with continuous-time volatility modeling. J. Bus. Econ. Stat. 30, 368-380.

Darrat, A.F., Rahman, S., Zhong, M., 2003. Intraday trading volume and return volatility of the DJIA stocks: A note. J. Bank. Finance 27, 2035-2043.

Diebold, F.X., Mariano, R.S., 1995. Comparing predictive accuracy. J. Bus. Econ. Stat. 13, $253-263$.

Donaldson, G., Kamstra, M., 2005. Volatility forecasts, trading volume, and the ARCH versus option-implied volatility trade-off. J. Fin. Res. 28, 519-538.

Fleming, J., Kirby, C., 2011. Long memory in volatiliy and trading volume. J. Bank. Finance $35,1714-1726$.

Fleming, J., Kirby, C., Ostdiek, B., 2003. The economic value of volatility timing using "realized" volatility. J. Fin. Econ. 67, 473-509.

Fuertes, A.-M., Izzeldin, M., Kalotychou, E., 2009. On forecasting daily stock volatility: The role of intraday information and market conditions. Int. J. Forecast. 25, 259-281.

Gallo, G.M., 2001. Modelling the impact of overnight surprises on intra-daily volatility. Australian Econ. Pap. 40, 567-580.

Gallo, G.M., Pacini, B., 2000. The effects of trading activity on market volatility. Eur. J. Finance 6, 163-175.

Ghysels, E., Santa-Clara, P., Valkanov, R., 2006. Predicting volatility: Getting the most out of return data sampled at different frequencies. J. Econometrics 131, 59-95. 
Hansen, P.R., Lunde, A., 2005. A realized variance for the whole day based on intermittent high-frequency data. J. Fin. Econometrics 3, 525-554.

Hansen, P.R., Lunde, A., 2006. Realized variance and market microstructure noise. J. Bus. Econ. Stat. 24, 127-218 (with comments and rejoinder).

Jacob, J., Vipul, 2008. Estimation and forecasting of stock volatility with range-based estimators. J. Fut. Market. 28, 561-581.

Jones, C.M., Kaul, G., Lipson, M.L., 1994. Transactions, volume, and volatility. Rev. Fin. Stud. $7,631-651$.

Koopman, S.J., Jungbacker, B., Hol, E., 2004. Forecasting daily variability of the S\&P 100 stock index using historical, realised and implied volatility measurements. J. Emp. Finance $12,445-475$.

Martens, M., 2002. Measuring and forecasting S\&P 500 index-futures volatility using highfrequency data. J. Fut. Market. 22, 497-518.

Martens, M., van Dijk, D., 2007. Measuring volatility with the realized range. J. Econometrics $138,181-207$.

McAleer, M., Medeiros, M., 2008. Realized volatilty: A review. Economet. Rev. 26, 10-45.

Müller, U., Dacorogna, M., Dav, R., Olsen, R., Pictet, O., Weizsacker, J.v., 1997. Intraday trading volume and return volatility of the DJIA stocks: A note. J. Bank. Finance 27, 20352043.

Naes, R., Skjeltorp, J.A., 2006. Order book characteristics and the volume-volatility relation: Empirical evidence from a limit order market. J. Fin. Market. 9, 408-432.

Park, B.-J., 1980. Surprising information, the MDH, and the relationship between volatility and trading volume. J. Fin. Market. 13, 344-366. 
Parkinson, M., 1980. The extreme value method for estimating the variance of the rate of return. J. Bus. 53, 61-65.

Rossi, E., Spazzini, F., 2009. Finite sample results of range-based integrated volatility estimation, CEA-Cass Working Paper Series, WP-CEA-04-2009.

Shu, J., Zhang, J.E., 2006. Testing range estimators of historical volatility. J. Fut. Market. 26, $297-313$.

Todorova, N., 2012. Volatility estimators based on daily price ranges versus the realized range. App. Fin. Econ. 22, 215-229.

Todorova, N., Husmann, S., 2012. A comparative study of range-based stock return volatility estimators for the German market. J. Fut. Market. 32, 560-586.

Tseng, T.-C., Lai, H.-C., Lin, C.-F., 2012. The impact of overnight returns on realized volatility. App. Fin. Econ. 22, 357-364.

Zhang, L., Mykland, P.A., Ait-Sahalia, Y., 2005. A tale of two time scales: Determining integrated volatility with noisy high-frequency data. J. Amer. Statist. Assoc. 100, 1394-1411. 


\section{Appendix: Tables}

Table 1: Descriptive statistics of the annualized daily realized ranges

\begin{tabular}{lcccccc}
\hline & Mean & SD & Skew & Kurt & Min & Max \\
\hline Merck & 0.2712 & 0.1128 & 1.3738 & 2.6463 & 0.0753 & 0.8231 \\
Fresenius & 0.2458 & 0.1245 & 1.8079 & 3.9195 & 0.0632 & 0.9451 \\
Henkel & 0.2087 & 0.0847 & 1.3673 & 2.5912 & 0.0514 & 0.6809 \\
Linde & 0.2381 & 0.1182 & 1.9317 & 4.3816 & 0.0727 & 0.8718 \\
Lufthansa & 0.2805 & 0.1159 & 1.8309 & 4.0017 & 0.1237 & 0.9256 \\
Metro & 0.2567 & 0.1322 & 1.9507 & 4.1621 & 0.0806 & 0.9850 \\
Adidas & 0.2176 & 0.0774 & 1.1716 & 1.7647 & 0.0661 & 0.5907 \\
MAN & 0.2957 & 0.1331 & 1.5352 & 2.9206 & 0.0975 & 0.9982 \\
Dt Post & 0.2550 & 0.1096 & 1.6788 & 3.3237 & 0.0993 & 0.8660 \\
Dt Börse & 0.2406 & 0.0915 & 1.3007 & 3.2270 & 0.0646 & 0.7828 \\
Continental & 0.2455 & 0.0933 & 1.8808 & 7.7579 & 0.0823 & 1.0882 \\
ThyssenKrupp & 0.2895 & 0.1280 & 1.4191 & 2.3029 & 0.1008 & 0.9818 \\
Infineon & 0.3268 & 0.1533 & 2.1779 & 5.8454 & 0.1237 & 1.3922 \\
Commerzbank & 0.2981 & 0.1492 & 1.8672 & 3.9204 & 0.1035 & 1.1405 \\
BMW & 0.2436 & 0.1054 & 2.0664 & 5.9258 & 0.0920 & 0.9736 \\
Bayer & 0.2730 & 0.1474 & 2.9616 & 14.7257 & 0.0983 & 1.7662 \\
RWE & 0.2452 & 0.1137 & 2.4037 & 7.7157 & 0.0905 & 0.9936 \\
Volkswagen & 0.2530 & 0.1123 & 2.0276 & 5.8363 & 0.1039 & 1.0209 \\
BASF & 0.2239 & 0.1028 & 2.0238 & 4.9590 & 0.0878 & 0.7961 \\
MRück & 0.2474 & 0.1444 & 2.2727 & 5.8581 & 0.0791 & 1.0328 \\
Dt Telekom & 0.2228 & 0.1085 & 2.6613 & 8.2224 & 0.1115 & 0.8928 \\
SAP & 0.2371 & 0.1015 & 1.8407 & 4.8580 & 0.0878 & 0.8843 \\
Daimler & 0.2632 & 0.1170 & 1.7609 & 3.4299 & 0.1068 & 0.8015 \\
Dt Bank & 0.2300 & 0.1078 & 2.0729 & 5.3355 & 0.0854 & 0.8434 \\
Siemens & 0.2356 & 0.1087 & 2.0010 & 4.6473 & 0.0974 & 0.7713 \\
Allianz & 0.2490 & 0.1450 & 2.2905 & 6.3322 & 0.0757 & 1.1309 \\
\hline & & & & & &
\end{tabular}


Table 2: Basic characteristics of the overnight returns, daily trading volume and number of transactions

\begin{tabular}{|c|c|c|c|c|c|c|c|c|}
\hline & \multicolumn{2}{|c|}{$\mathrm{R}$} & \multicolumn{2}{|c|}{ RABS } & \multicolumn{2}{|c|}{ VOL } & \multicolumn{2}{|c|}{ NTR } \\
\hline & Mean & SD & Mean & SD & Mean & SD & Mean & SD \\
\hline Merck & 0.0162 & 9981 & 6752 & 0.7349 & 372900 & 373942 & 980 & 878 \\
\hline Fres & 0170 & 307 & 4575 & 1343 & 4651 & 554 & 1244 & $c \rho$ \\
\hline Henkel & 0.0272 & 0.6752 & 4421 & 0.5109 & 516590 & 49637 & 1257 & 767 \\
\hline Linde & 0.0238 & 0.6653 & 0.4857 & 0.4552 & 704435 & 461926 & 1439 & 38 \\
\hline Luftha & 0.0038 & & 0.5809 & & 3698277 & 1909216 & 1567 & 692 \\
\hline & 1 & 7 & 5512 & & 330 & & 1639 & 774 \\
\hline A & 9 & 4 & 4794 & 0 & 75 & 118 & 1726 & 1004 \\
\hline MAN & 1146 & 0.9652 & 0.6811 & 0.6932 & 1088855 & 782169 & 1759 & 1223 \\
\hline Dt Pos & 0297 & 0.7008 & 0.4926 & 0.4991 & 4051631 & 3139502 & 1811 & 949 \\
\hline Dt E & 0.0738 & 0.8027 & 0.5506 & 0.58 & 939290 & & 1829 & 1346 \\
\hline Cont & & & & & & & 95 & 1334 \\
\hline & & & & & 91 & & 2021 & 1198 \\
\hline Infi & 0.1352 & 1 & 0.8910 & 0.8 & 1023 & & 2236 & 1058 \\
\hline Comr & 0.0969 & 0.9416 & 0.6749 & 0.6634 & 4578200 & 70 & 2369 & 1533 \\
\hline BMI & 0.0734 & - & 0.5347 & $a_{-1}$ & 48 & 7 & 2536 & 1123 \\
\hline Bayer & 0.0646 & 0.8650 & 0.5955 & 0.6305 & 4867442 & 2684865 & 3252 & 1666 \\
\hline RWE & & & & & & & 3280 & 1762 \\
\hline Volk & 00 & & & & & & 3284 & 1944 \\
\hline $\mathrm{BAS}$ & 1 & 0 & 90 & & & 128 & 3431 & 1458 \\
\hline IR & 4 & 0.93 & 0.6419 & 0 & 75 & 1013 & 3935 & 1636 \\
\hline $\mathrm{Dt}$ ? & 8 & 0.7245 & 0.4614 & 0.5592 & 25388140 & 13217630 & 4019 & 1406 \\
\hline SAP & 0.0278 & 0.9250 & 0.6344 & 0.6735 & 3209937 & 4127521 & 4302 & 2128 \\
\hline Daiml & 0233 & 0.8737 & 0.6109 & 0.6248 & 6659873 & 4198058 & 4808 & 2768 \\
\hline Dt Be & 0311 & 0.0 & 0.5997 & & 70 & 2453770 & 5037 & 2641 \\
\hline & & & & & & 2956 & 5636 & 2850 \\
\hline Allic & 0.0864 & 1.0224 & 0.6923 & 0.7571 & 3338192 & 1711656 & 6020 & 2827 \\
\hline
\end{tabular}

Notes: The sample period extends from 2003 to 2007 with 1268 trading days in total. The overnight return $R$ and the absolute overnight return $R A B S$ are given in per cent. $V O L$ contains the sum of all stocks traded during the official open market time per day and $N T R$ labels the corresponding daily number of transactions. 
Table 3: Lead-lag correlations between the daily realized range with overnight returns, daily stock volume and number of trades

\begin{tabular}{|c|c|c|c|c|c|}
\hline & $\mathrm{R}$ & RNEG & RABS & VOL & NTR \\
\hline Merck & $-0.1001^{* * *}$ & $0.3224^{* * *}$ & $-0.2620 * * *$ & $0.0720 * *$ & 0.0362 \\
\hline Fresenius & $-0.0650^{* *}$ & $0.2835^{* * *}$ & $-0.2084^{* * *}$ & $0.1528 * * *$ & $0.2061^{* * *}$ \\
\hline Henkel & -0.0191 & $0.2884^{* * *}$ & $-0.1959 * * *$ & $0.3062^{* * *}$ & $0.3195^{* * *}$ \\
\hline Linde & $-0.0564^{* *}$ & $0.2496^{* * *}$ & $-0.1800 * * *$ & 0.0311 & 0.0316 \\
\hline Lufthansa & $-0.0700 * *$ & $0.3872^{* * *}$ & $-0.2740 * * *$ & 0.0426 & $0.1932^{* * *}$ \\
\hline Metro & $0.0542^{*}$ & $0.3901 * * *$ & $-0.1860 * * *$ & $0.0811^{* * *}$ & $0.0616^{* *}$ \\
\hline Adidas & $-0.1787^{* * *}$ & $0.3460^{* * *}$ & $-0.3266^{* * *}$ & $0.2374^{* * *}$ & $0.2350^{* * *}$ \\
\hline MAN & -0.0405 & $0.3234^{* * *}$ & $-0.2362^{* * *}$ & $0.1058 * * *$ & $0.1133^{* * *}$ \\
\hline Dt Post & -0.0293 & $0.2498 * * *$ & $-0.1560 * * *$ & $-0.1041^{* * *}$ & 0.0044 \\
\hline Dt Börse & -0.0416 & $0.2399 * * *$ & $-0.1687^{* * *}$ & $0.2498^{* * *}$ & $0.3429^{* * *}$ \\
\hline Continental & $-0.0625^{* *}$ & $0.2598^{* * *}$ & $-0.2032^{* * *}$ & $0.1135^{* * *}$ & $0.0710^{* *}$ \\
\hline ThyssenKrupp & -0.0403 & $0.3231^{* * *}$ & $-0.2201^{* * *}$ & $0.0689^{* *}$ & $0.1412^{* * *}$ \\
\hline Infineon & -0.0218 & $0.4052^{* * *}$ & $-0.2537^{* * *}$ & $0.1257 * * *$ & $0.4942^{* * *}$ \\
\hline Commerzbank & -0.0204 & $0.3805^{* * *}$ & $-0.2440^{* * *}$ & $0.0855^{* * *}$ & $0.0809^{* * *}$ \\
\hline BMW & $-0.0479^{*}$ & $0.3345^{* * *}$ & $-0.2405^{* * *}$ & $0.1783^{* * *}$ & $0.2698^{* * *}$ \\
\hline Bayer & 0.0040 & $0.3384^{* * *}$ & $-0.2038 * * *$ & $0.3241 * * *$ & $0.2714^{* * *}$ \\
\hline RWE & $-0.1213^{* * *}$ & $0.2614^{* * *}$ & $-0.2279 * * *$ & $0.0595^{* * *}$ & -0.0041 \\
\hline Volkswagen & $-0.0704^{* *}$ & $0.2605^{* * *}$ & $-0.1866 * * *$ & $0.1407 * * *$ & $0.2065^{* * *}$ \\
\hline BASF & $-0.0728^{* * *}$ & $0.2923^{* * *}$ & $-0.2348^{* * *}$ & $0.3198 * * *$ & $0.2790^{* * *}$ \\
\hline MRück & $-0.1123^{* * *}$ & $0.4544^{* * *}$ & $-0.3587 * * *$ & $0.2508^{* * *}$ & $0.4267^{* * *}$ \\
\hline Dt Telekom & -0.0435 & $0.3774^{* * *}$ & $-0.2303^{* * *}$ & $0.1251^{* * *}$ & $0.5097^{* * *}$ \\
\hline SAP & $-0.1591^{* * *}$ & $0.3017 * * *$ & $-0.2849 * * *$ & $0.1456^{* * *}$ & $0.2156^{* * *}$ \\
\hline Daimler & $-0.0835^{* * *}$ & $0.2547 * * *$ & $-0.2177^{* * *}$ & $0.1613^{* * *}$ & $0.2846^{* * *}$ \\
\hline Dt Bank & $-0.1001^{* * *}$ & $0.3042^{* * *}$ & $-0.2437 * * *$ & $0.3303^{* * *}$ & $0.2739^{* * *}$ \\
\hline Siemens & $-0.0881^{* * *}$ & $0.2666^{* * *}$ & $-0.2283^{* * *}$ & $0.2933^{* * *}$ & $0.3090^{* * *}$ \\
\hline Allianz & $-0.0685^{* *}$ & $0.4427 * * *$ & $-0.3259 * * *$ & $0.3168^{* * *}$ & $0.3518^{* * *}$ \\
\hline
\end{tabular}

Notes: Correlations are presented for the daily realized range of day $t, R R_{t}$ with the immediately preceding overnight return $R_{t-1, t}$, absolute overnight return $R A B S_{t-1, t}$ and the leveraged specification of the overnight return $R N E G_{t-1, t}$ which considers negative values of overnight returns only. The last two columns present the correlation of $R R_{t}$ to the amount of traded stocks and the number of trades of the previous day, $t-1$.

$*, * *$ and $* * *$ indicate significance at the 10,5 and $1 \%$ levels, respectively. 


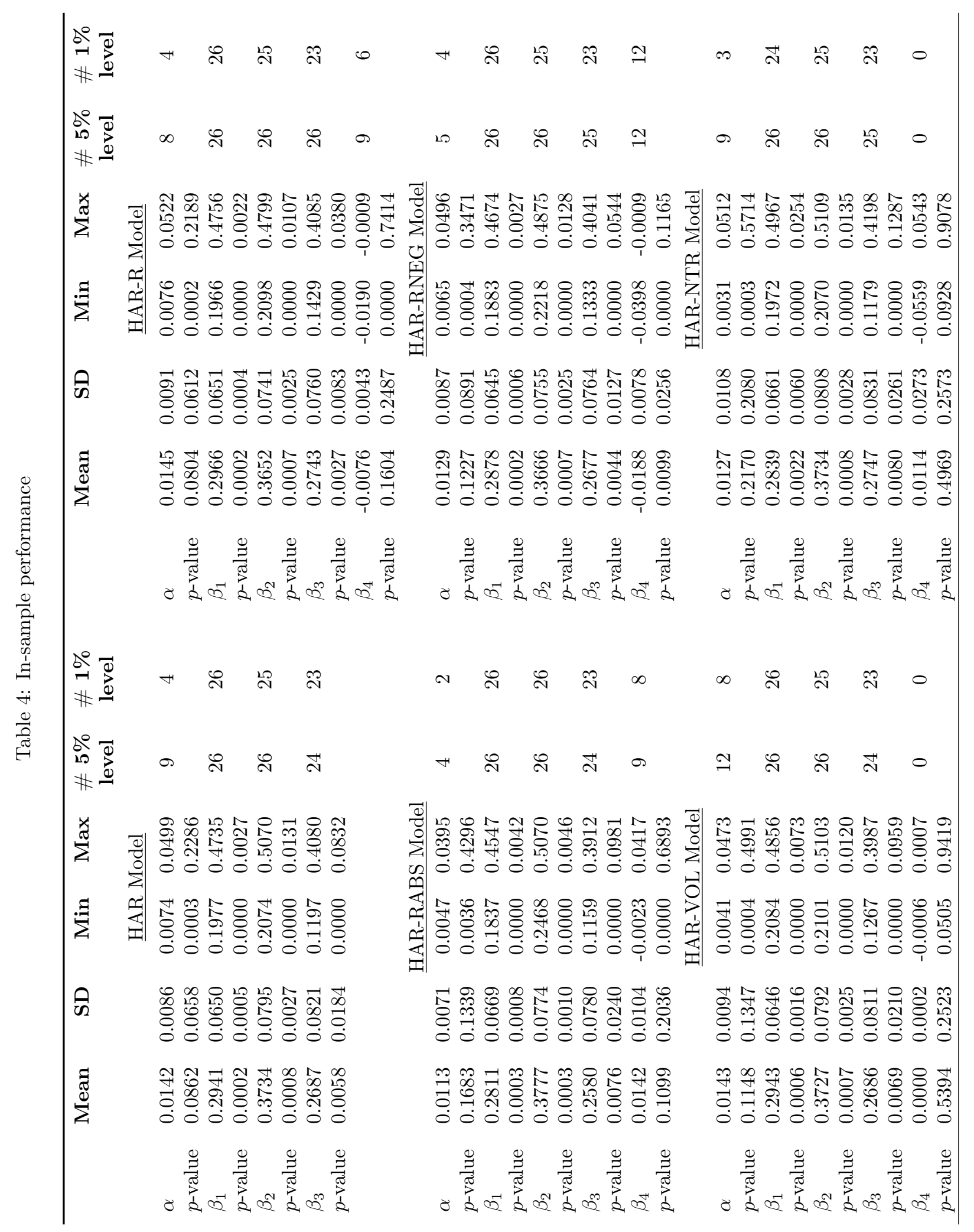

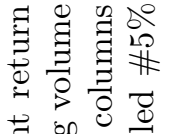
然国 桪 5 蛋完 象 a. 我 - 0 \% 政 递专 品

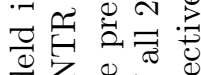

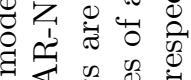

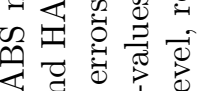
पद चี

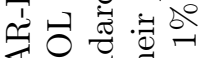
还只 表

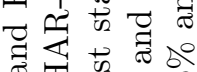
政考

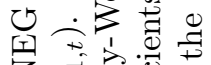
证获

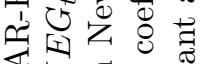
出

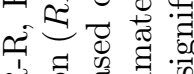

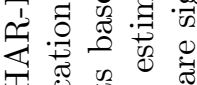

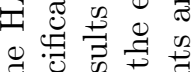

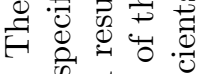

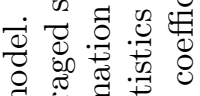

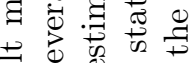

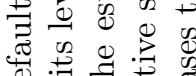
尊 चै

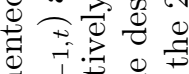
焉古焉 चै

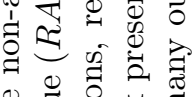

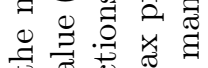

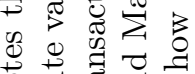

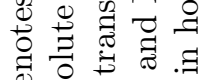
क्ष

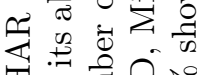

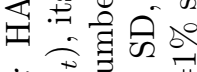
\% 之े 
Table 5: Adjusted $R^{2}$ of the in-sample regressions

\begin{tabular}{lcccccc}
\hline & HAR & HAR-R & HAR-RNEG & HAR-RABS & HAR-VOL & HAR-NTR \\
\hline Merck & 0.2379 & 0.2447 & 0.3088 & 0.2850 & 0.2373 & 0.2371 \\
Fresenius & 0.7503 & 0.7507 & 0.7548 & 0.7532 & 0.7501 & 0.7504 \\
Henkel & 0.5724 & 0.5721 & 0.5873 & 0.5806 & 0.5720 & 0.5720 \\
Linde & 0.7134 & 0.7132 & 0.7163 & 0.7144 & 0.7132 & 0.7132 \\
Lufthansa & 0.7458 & 0.7486 & 0.7539 & 0.7548 & 0.7455 & 0.7458 \\
Metro & 0.4659 & 0.4970 & 0.5371 & 0.5357 & 0.4662 & 0.4669 \\
Adidas & 0.7466 & 0.7479 & 0.7547 & 0.7526 & 0.7468 & 0.7467 \\
MAN & 0.8191 & 0.8191 & 0.8221 & 0.8206 & 0.8191 & 0.8190 \\
Dt Post & 0.5177 & 0.5176 & 0.5267 & 0.5224 & 0.5173 & 0.5181 \\
Dt Börse & 0.6837 & 0.6842 & 0.6927 & 0.6901 & 0.6836 & 0.6839 \\
Continental & 0.4140 & 0.4185 & 0.4229 & 0.4268 & 0.4148 & 0.4150 \\
ThyssenKrupp & 0.7494 & 0.7494 & 0.7564 & 0.7533 & 0.7491 & 0.7492 \\
Infineon & 0.7691 & 0.7762 & 0.7751 & 0.7820 & 0.7692 & 0.7696 \\
Commerzbank & 0.7917 & 0.7933 & 0.7939 & 0.7957 & 0.7915 & 0.7915 \\
BMW & 0.7758 & 0.7845 & 0.7760 & 0.7848 & 0.7757 & 0.7756 \\
Bayer & 0.8446 & 0.8484 & 0.8463 & 0.8494 & 0.8445 & 0.8445 \\
RWE & 0.6711 & 0.6734 & 0.6724 & 0.6743 & 0.6710 & 0.6710 \\
Volkswagen & 0.8123 & 0.8129 & 0.8175 & 0.8166 & 0.8122 & 0.8123 \\
BASF & 0.8243 & 0.8281 & 0.8246 & 0.8285 & 0.8246 & 0.8243 \\
MRück & 0.8757 & 0.8828 & 0.8757 & 0.8800 & 0.8756 & 0.8758 \\
Dt Telekom & 0.8819 & 0.8858 & 0.8865 & 0.8882 & 0.8818 & 0.8821 \\
SAP & 0.7513 & 0.7546 & 0.7523 & 0.7556 & 0.7523 & 0.7512 \\
Daimler & 0.6817 & 0.6830 & 0.6826 & 0.6828 & 0.6815 & 0.6813 \\
Dt Bank & 0.8344 & 0.8384 & 0.8343 & 0.8375 & 0.8344 & 0.8344 \\
Siemens & 0.8322 & 0.8380 & 0.8326 & 0.8381 & 0.8326 & 0.8326 \\
Allianz & 0.8612 & 0.8657 & 0.8611 & 0.8640 & 0.8612 & 0.8611 \\
\hline
\end{tabular}

Notes: The basic form of the estimated model is

$$
R R_{t}=\alpha+\beta_{1} R R_{t-1}+\beta_{2} R R_{t-5, t-1}+\beta_{3} R R_{t-21, t-1}+\beta_{4} X_{t-1}+\omega_{t} .
$$

The variable $X_{t-1}$ is set to zero for the HAR model. The HAR-R, HAR-RNEG and HAR-RABS model incorporate the previous overnight return $\left(R_{t-1, t}\right)$, its absolute value $\left(R A B S_{t-1, t}\right)$ and its leveraged specification $\left(R N E G_{t-1, t}\right)$, respectively. HAR-VOL and HAR-NTR comprise the previous days' trading volume, divided by 1000000 and number of transactions, divided by 10000 , respectively. 


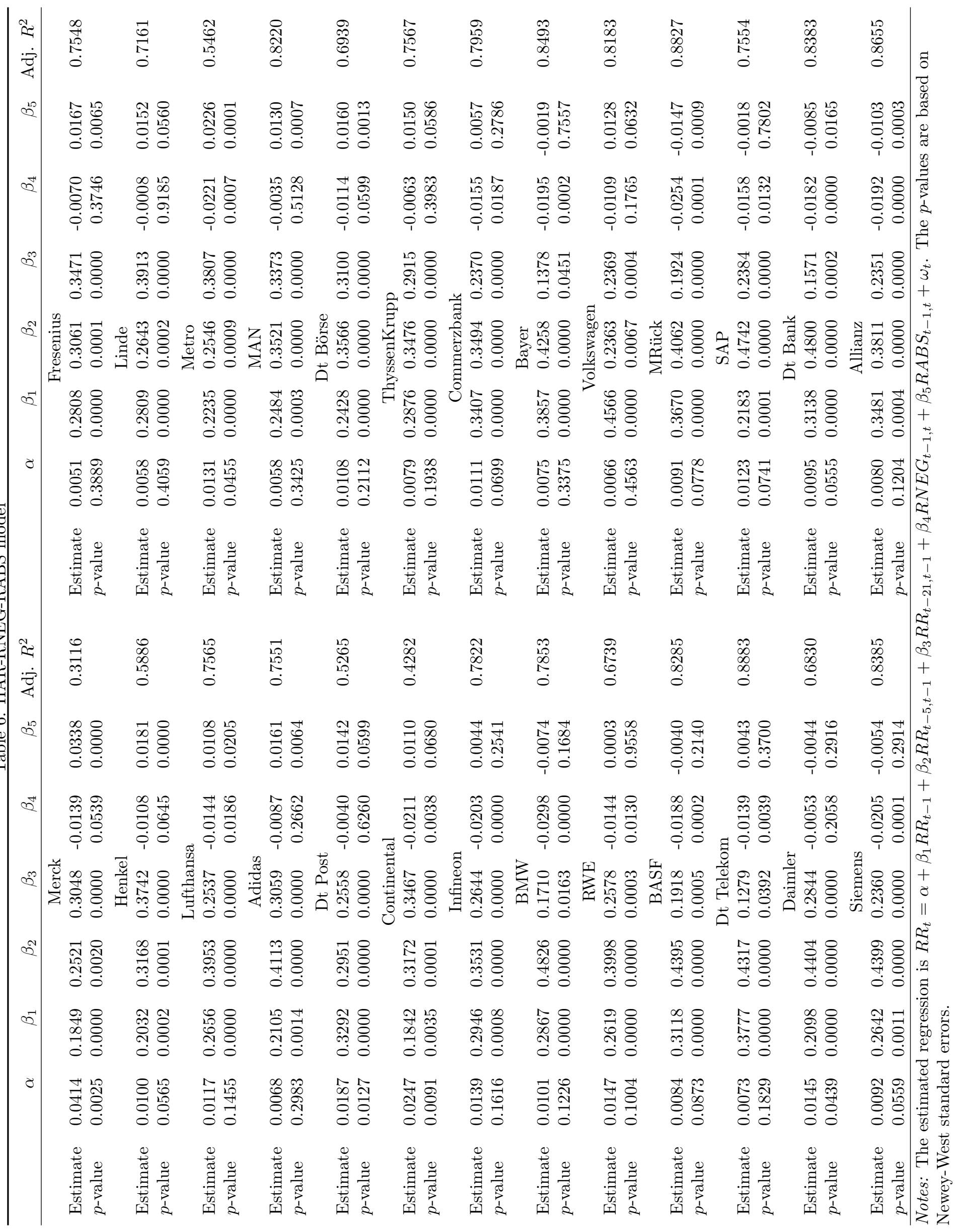



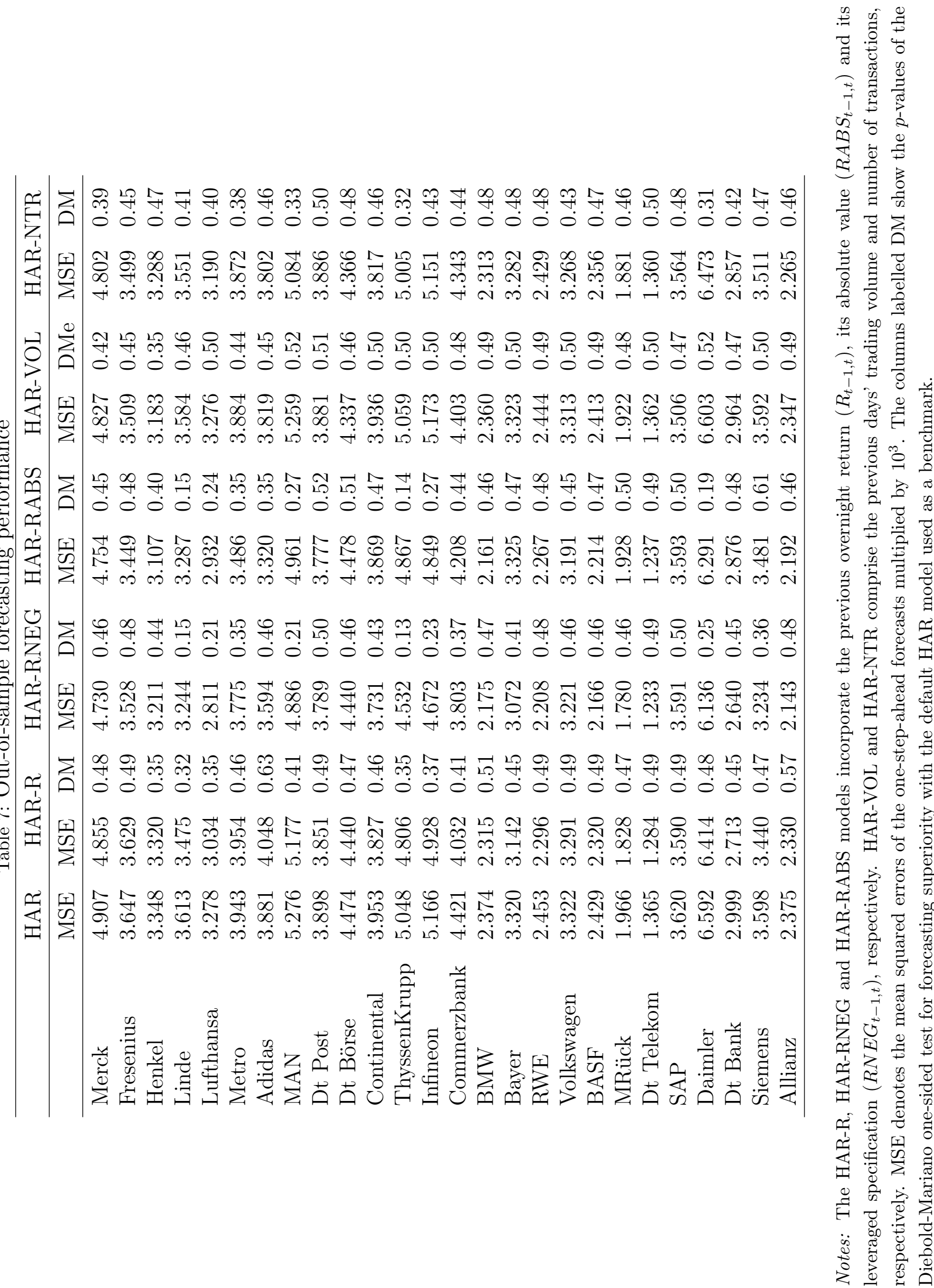